\title{
Tongue Laceration during Neurophysiologic Monitoring with Motor Evoked Potentials
}

\section{Sergey Pisklakov, Vanny Le, Paul Sandoval}

Department of Anesthesiology and Perioperative Medicine, University of Medicine and Dentistry of New Jersey, New Jersey Medical School, Newark, USA.

Email: pisklase@umdnj.edu

Received September 19 $9^{\text {th }}$ 2012; revised October 19 ${ }^{\text {th }}$ 2012; accepted October $29^{\text {th }}, 2012$

\begin{abstract}
Although considered a safe modality for monitoring spinal cord function, motor evoked potentials (MEP) may cause tongue laceration in rare cases. Bite blocks are essential in order to prevent tongue and mucosal injury during monitoring with MEP, but there is no consensus on the ideal bite block to use for this purpose. Potential concerns include tongue necrosis from prolonged use of an oral airway as a hard bite block, and TMJ dysfunction caused by occlusal interference when blocks interrupt the normal occlusal surface. We had a case of tongue laceration during MEPs. Bite blocks should be placed prior to the start of monitoring to prevent teeth occlusion. Because rigid bite blocks may cause pressure injury to oropharyngeal structures soft bite blocks are recommended. The bite block needs to be placed in a way to prevent occlusion by both front teeth and molars and to keep the tongue in the middle of the mouth. Ongoing vigilance to bite block placement is strongly recommended.
\end{abstract}

Keywords: Neuromonitoring; Airway Injury; Bite Blocks

\section{Introduction}

Somato-sensory evoked potentials (SSEPs) continue to be the most frequently used intraoperative monitoring method to assess the integrity of the spinal dorsal column but, cannot be relied on to directly monitor motor function. The simultaneous monitoring of SSEPs and motor evoked potentials (MEPs) offers superior detection of spinal cord injury [1]. Although considered a safe modality for monitoring spinal cord function, MEPs have been known to cause tongue laceration in rare cases [2].

\section{Case}

A 28-year-old woman who previously underwent a T4 T7 laminectomy for an intradural mass lesion presented with a three month history of weakness progressing to paraparesis and loss of normal bowel and bladder function. Evaluation of the patient revealed progressive neurologic dysfunction secondary to a large intradural mass lesion and significant spinal cord compression. On physical exam, she had a Mallampati Class II airway and both upper and lower front teeth were intact. The patient was scheduled for a decompression, stabilization, and fusion of the thoracic spine with SSEPs and MEPs monitoring requested by the surgeon. Induction and intubation were uneventful. An oral airway was inserted as a bite block to prevent biting of the tongue and oral mucosa during monitoring of MEPs. The patient underwent extensive surgery to remove the intradural mass lesion under total intravenous anesthesia. Before emergence, a small amount of blood was noted on the bite block. Suctioning revealed more blood in the mouth. A $3 \mathrm{~cm}$ laceration of the ventral surface of her tongue was noted. We concluded that the laceration was a result of stimulation of the masseter muscle during monitoring with MEPs. An ENT consult was called and the laceration was sutured with four absorbable sutures. Subsequent emergence and extubation were uneventful. On following postoperative day, the patient noted mild tongue soreness and difficulty swallowing. However, she was discharged from the hospital as scheduled and recovered uneventfully.

\section{Discussion and Conclusions}

MEP monitoring is a well-established effective method to monitor the integrity of neurologic pathways during spine surgery. MEP monitoring generally involves subcutaneous electrodes inserted into the scalp that apply between 300 - $1000 \mathrm{~V}$ and generate up to $1500 \mathrm{~mA}$ bursts across cortical area. This intensive stimulation produces excitation of the corticospinal tract neurons, and ultimately stimulation of the distal muscle. 
MEP monitoring is generally safe. Retrospective reviews [3,4] report complications such as seizures, skin burns and arrhythmias were most common. Tongue laceration is overall a rare complication of neurophysiologic monitoring with motor evoked potentials [2]. The incidence of tongue and lip injuries (due to evoked-potentialinduced jaw muscle activity) is estimated at 0.14 to 0.19 percent $[3,4,6]$ and case reports of oral airway trauma due to muscle activity have ranged from lip and tongue lacerations to mandibular fracture. Prone positioning predisposes to tongue swelling and increase the chance of the tongue being caught between the teeth [5]. A. Tamkus and K. Rice recently reviewed the incident reports of MEP-associated bite injuries from 17,273 consecutive surgical procedures. Bite injuries occurred with an incidence of $0.63 \%$, the most severe of which requiring sutures at an incidence of $0.14 \%$. Injuries of the tongue occurred approximately four times as frequently as injuries of the lip [6]. Most clinicians would place a bite block to separate the upper and lower teeth from each other and to keep the tongue from protruding between the upper and lower jaw. However, this does not prevent lacerations or other oropharyngeal injury. There are a significant number of case reports of bite block dislodgement, movement of the tongue between the teeth and subsequent injury [2,4]. Most reviews recommend a soft block to prevent trauma from repeated biting. Nevertheless, soft blocks are known to deteriorate under the force of bite. Rigid bite blocks can cause prolonged pressure on the posterior tongue, causing lingual injury. Some clinicians report success with commercial products originally intended for dental care [4]. Frequently checking the position of the bite block and tongue to verify that the tongue remains protected is also recommended but, may be difficult because of the surgical field proximity.

Bite blocks are essential in order to prevent tongue and mucosal injury during monitoring with MEP, but there is no consensus on the ideal bite block to use for this pur- pose. Potential concerns include tongue necrosis from prolonged use of an oral airway as a hard bite block, and TMJ dysfunction caused by occlusal interference when blocks interrupt the normal occlusal surface [3]. However, these potential concerns have not been adequately verified. Bite blocks should be placed prior to the start of monitoring to prevent teeth occlusion. Because rigid bite blocks may cause pressure injury to oropharyngeal structures soft bite blocks are recommended. The bite block needs to be placed in a way to prevent occlusion by both front teeth and molars and to keep the tongue in the middle of the mouth. Ongoing vigilance to bite block placement is strongly recommended.

\section{REFERENCES}

[1] N. Malhotra and C. Shaffrey, "Intraoperative Electrophysiologic Monitoring during Spine Surgery,” Spine, Vol. 35, No. 25, 2010, pp. 2167-2179. doi:10.1097/BRS.0b013e3181f6f0d0

[2] D. Schwartz, et al., "Transcranial Electric Motor Evoked Potentials Monitoting for Spinal Surgery, Is It Safe?” Spine, Vol. 36, No. 13, 2011, pp. 1046-1049. doi:10.1097/BRS.0b013e3181ecbe77

[3] D. MacDonald, "Intraoperative Motor Evoked Potential Monitoring: Overview and Update," Journal of Clinical Monitoring and Computing, Vol. 20, No. 5, 2006, pp. 347377. doi:10.1007/s10877-006-9033-0

[4] S. Deiner and I. Osborn, "Prevention of Airway Injury during Spine Surgery: Rethinking Bite Blocks,” Journal of Neurosurgical Anesthesiology, Vol. 21, No. 1, 2009, pp. 68-69. doi:10.1097/ANA.0b013e31818d205e

[5] A. Lam and M. Vavilala, "Macroglossia: Compartment Syndrome of the Tongue?” Anesthesiology, Vol. 92, No. 6, 2000, pp. 1832-1835. doi:10.1201/9780849376276

[6] A. Tamkus and K. Rice, “The Incidence of Bite Injuries Associated with Transcranial Motor-Evoked Potential Monitoring," Anesthesia \& Analgesia, Vol. 115, No. 3, 2012, pp. 663-667. 